\title{
Caracterização das camadas formadas pela nitretação a plasma em amostras de ferro sinterizado com e sem tratamento de ferroxidação
}

Characterization of plasma nitrided layers formed on sintered iron with and without steam treatment

Marcos Alves Fontes ${ }^{1}$, Ricardo G. Pereira ${ }^{2}$, Frederico A. P. Fernandes², Luiz C. Casteletti², Pedro A. P. Nascente

\section{RESUMO}

Nitretação a plasma é um processo de tratamento termofísico-químico que consiste em um endurecimento superficial, provocado pela difusão intersticial de nitrogênio atômico nas superfícies de ligas metálicas. Neste trabalho, este processo foi empregado na modificação superficial de uma liga de ferro sinterizado. Foram feitos análises via microscopia eletrônica de varredura (SEM ou MEV) e difração de raios X (XRD ou DRX) em amostras submetidas ao tratamento de ferroxidação e ao tratamento a plasma, realizado sob diferentes condições de tempo e temperatura. Os resultados mostraram que a espessura da camada de nitreto formada é maior para todas as amostras nitretadas em comparação à amostra ferroxidada, e a mesma aumenta com o tempo e temperatura de nitretação, sendo a temperatura a variável que mais influencia. Também, resultados identificaram que a camada nitretada, para todas as amostras analisadas, próxima à região superficial, consiste de uma mistura de fases de $\gamma^{\prime}-\mathrm{Fe}_{4} \mathrm{~N}$ e $\varepsilon-\mathrm{Fe}_{3} \mathrm{~N}$.

Palavras-chave: Nitretação iônica; Metalurgia do pó; Microscopia eletrônica de varredura; Difração de raios X.

\section{ABSTRACT}

Plasma nitriding is a thermo-physical-chemical treatment process which consists in a surface hardening, caused by the interstitia diffusion of atomic nitrogen into the metallic alloys' surfaces. In this work, this process was employed in the surface modification of a sintered ferrous alloy. Scanning electron microscopy (SEM) and $X$ ray diffraction $(\mathrm{XRD})$ analysis were performed on the samples submitted to steam treatment and plasma nitriding performed under different conditions of time and temperature. The results showed that the nitride layer thickness is higher for all nitrided samples than steam treatment samples, and this layer thickness increases with nitriding time and temperature, where the temperature is the variable that most influences. The XRD analysis showed that the nitrided layer, for all samples, near to the surface consists in a mixture of $\gamma^{\prime}-\mathrm{Fe}_{4} \mathrm{~N}$ e $\varepsilon$ - $\mathrm{Fe}_{3} \mathrm{~N}$ phases.

Keywords: Plasma nitriding; Powder sintered metal; Scanning electron microscopy; X-ray diffraction.

\footnotetext{
'Universidade Federal de São Carlos - São Carlos (SP) - Brasil

2Universidade de São Paulo - São Carlos (SP) - Brasil

Autor correspondente: Marcos A. Fontes - Universidade Federal de São Carlos - Rodovia Washington Luís, Km 235, s/n - Jardim Guanabara CEP: 13565-905 - São Carlos (SP) - Brasil

E-mail: fontesma@hotmail.com

Recebido: 02/08/2012 Aprovado: 24/02/2015
} 


\section{INTRODUÇÃO}

Vários tratamentos térmicos têm sido utilizados para melhorar as propriedades das superfícies dos materiais, como dureza, resistência ao desgaste e resistência à corrosão. A nitretação é um dos processos de tratamento superficial mais frequentemente encontrados para se obter essas melhorias, pois é considerado um processo simples, com baixo custo relativo, e boa capacidade de melhorar substancialmente a dureza das superfícies, bem como as resistências ao desgaste e corrosã $0^{(1)}$.

Dentre os três diferentes tipos de processos de nitretação, líquida, gasosa e a plasma (ou iônica), a última se destaca por possuir algumas características positivas como: controle preciso das fases das camadas da superfície nitretada, baixo consumo de energia e gás, não gerar poluição ambiental, e possibilitar o tratamento térmico a baixas temperaturas, acima de $350^{\circ} \mathrm{C}^{(1)}$.

A nitretação iônica é um processo de tratamento termo-físico-químico que consiste em um endurecimento superficial, provocado pela difusão intersticial de nitrogênio atômico na superfície de ligas ferrosas e não ferrosas ${ }^{(2)}$. Esta difusão leva a formação de uma superfície modificada que pode ser constituída por uma camada de óxido, uma camada composta e uma zona de difusão ${ }^{(3-6)}$. Para as ligas ferrosas, são encontradas duas camadas superficiais, sendo que a primeira é denominada camada composta, ou camada branca, e é formada por nitretos de ferro dos tipos $\mathrm{Fe}_{3} \mathrm{~N}(\varepsilon), \mathrm{Fe}_{4} \mathrm{~N}\left(\gamma^{\prime}\right)$ e, talvez, $\mathrm{Fe}_{2} \mathrm{~N}^{(7-11)}$. A segunda camada, chamada de zona de difusão, ou camada de difusão, é formada entre a camada composta e a matriz e é constituída por nitretos formados devido à reação do nitrogênio com o ferro e também com metais de maior afinidade, tais como alumínio, cromo, vanádio e molibdênio ${ }^{(11)}$.

A nitretação a plasma proporciona camadas nitretadas com espessuras uniformes e mais facilmente controladas, e são bastante indicadas para materiais sinterizados. Para melhores desempenhos de serviço em aplicações de engenharia, é crucial o controle da formação e espessura das camadas nitretadas, as quais podem ser alcançadas pelo controle dos parâmetros de nitretação, e esta versatilidade é a primeira das principais características deste processo. As espessuras e as composições das camadas nitretadas são funções da temperatura e do tempo de tratamento, bem como da composição do material base ${ }^{(12-15)}$.

A difusividade dos átomos de nitrogênio na estrutura do ferro varia com a temperatura de tratamento por meio da seguinte equação ${ }^{(16-18)}$ :

$\mathrm{D}=6,6 \times 10^{-7} \times \exp (-77.900 / \mathrm{R} \times \mathrm{T})$

onde: $\mathrm{R}=$ constante universal dos gases e $\mathrm{T}=$ temperatura $\mathrm{ab}$ soluta $[\mathrm{K}]$

O objetivo deste trabalho é caracterizar as amostras nitretadas ionicamente, por meio da comparação das espessuras das camadas nitretadas formadas para cada condição de nitretação (diferentes tempos e temperaturas de tratamento), com amostras submetidas ao tratamento superficial de ferroxidação, como também amostras sem tratamento nenhum, além da avaliação das fases presentes após o processo de tratamento superficial.

A liga de ferro escolhida para a avaliação é utilizada na fabricação de um componente de compressores para refrigeração, e o aumento da sua robustez permite aumentar a gama de aplicações dos produtos.

\section{PROCEDIMENTOS EXPERIMENTAIS}

As amostras de ferro sinterizado, cuja composição química está apresentada na Tabela 1, foram preparadas inicialmente por meio de lixamento, usando progressivamente lixas com granulação referente aos números 220, 320, 400, 500, 600 e 800 e sequencialmente polidas em uma politriz com alumina $0,05 \mu \mathrm{m}$. Após o polimento, as amostras foram lavadas em água e álcool, secas e armazenadas. Antes de serem introduzidas no dispositivo de nitretação, as amostras foram lavadas com tetracloroetileno em ultra-som durante 60 minutos, para eliminação de gorduras e outras impurezas superficiais.

Tabela 1: Composição química da amostra de liga ferrosa.

\begin{tabular}{c|c|}
\hline Elemento & $\%$ em peso \\
\hline Carbono combinado & $0,20-0,40$ \\
Cobre & $1,75-2,25$ \\
Enxofre & $0,14-0,22$ \\
\hline Ferro & Restante \\
\hline
\end{tabular}

Após serem colocadas no dispositivo de nitretação, as superfícies das amostras foram limpas utilizando-se o processo de pulverização catódica (sputtering), sob uma pressão de $500 \mathrm{~Pa}$, a uma temperatura $50^{\circ} \mathrm{C}$ menor que a temperatura de nitretação a plasma, por 60 minutos. Para o processo de nitretação a plasma, realizado em um reator de fonte de alta tensão pulsada, utilizou-se

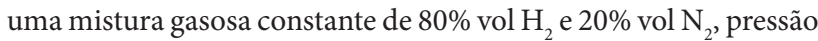
de $500 \mathrm{~Pa}$, temperaturas de nitretação de $500^{\circ} \mathrm{C}, 540^{\circ} \mathrm{C}$ e $580^{\circ} \mathrm{C}$ e períodos de tratamento de 3 e 5 horas. A medida da temperatura foi feita por meio de um termopar inserido no porta-amostra. Fernandes et al. ${ }^{(19)}$ fornece mais detalhes sobre o equipamento.

Para a análise por MEV, utilizou-se um microscópio da Philips modelo SL-30 com canhão de emissão por campo, equipado com um espectrômetro de energia dispersiva (EDS). As amostras embutidas foram previamente preparadas e recobertas com ouro e prata para permitir contato elétrico entre a amostra e o suporte da câmara. As imagens foram adquiridas empregando-se elétrons retroespalhados (BSE), que permitem a visualização de diferenças na composição química das amostras.

As análises de DRX foram realizadas utilizando-se o equipamento de raios X Rigaku modelo Geiger-Flex, sob as seguintes condições: radiação $\mathrm{Cu}-\mathrm{Ka}$, com comprimento de onda $\lambda=1,54056 \AA$; ângulo de varredura $(\theta-2 \theta)$ de $5^{\circ}$ a $90^{\circ}$; ângulo de avanço: $0,032^{\circ}$ segundo. Para a identificação das fases e a caracterização dos picos de difração, utilizou-se o programa Diffrac EVA Release 2001. 


\section{RESULTADOS E DISCUSSÃO Análise via MEV}

A análise via MEV foi efetiva para obter a espessura tanto da camada de ferrox bem como a espessura da camada de todas as amostras nitretadas. Observa-se visualmente, nas imagens apresentadas na Fig. 1, que as amostras nitretadas com temperaturas de 540 e $580^{\circ} \mathrm{C}$ para as duas condições de tempo de nitretação, apresentaram uma maior penetração de nitrogênio, promovendo uma maior espessura de camada nitretada. Além da formação de uma camada de nitreto nas superfícies das amostras, têm-se uma grande quantidade de nitrogênio no interior da matriz, mostrando que independentemente do tempo de exposição na nitretação, as altas temperaturas favorecem a difusão do nitrogênio para o

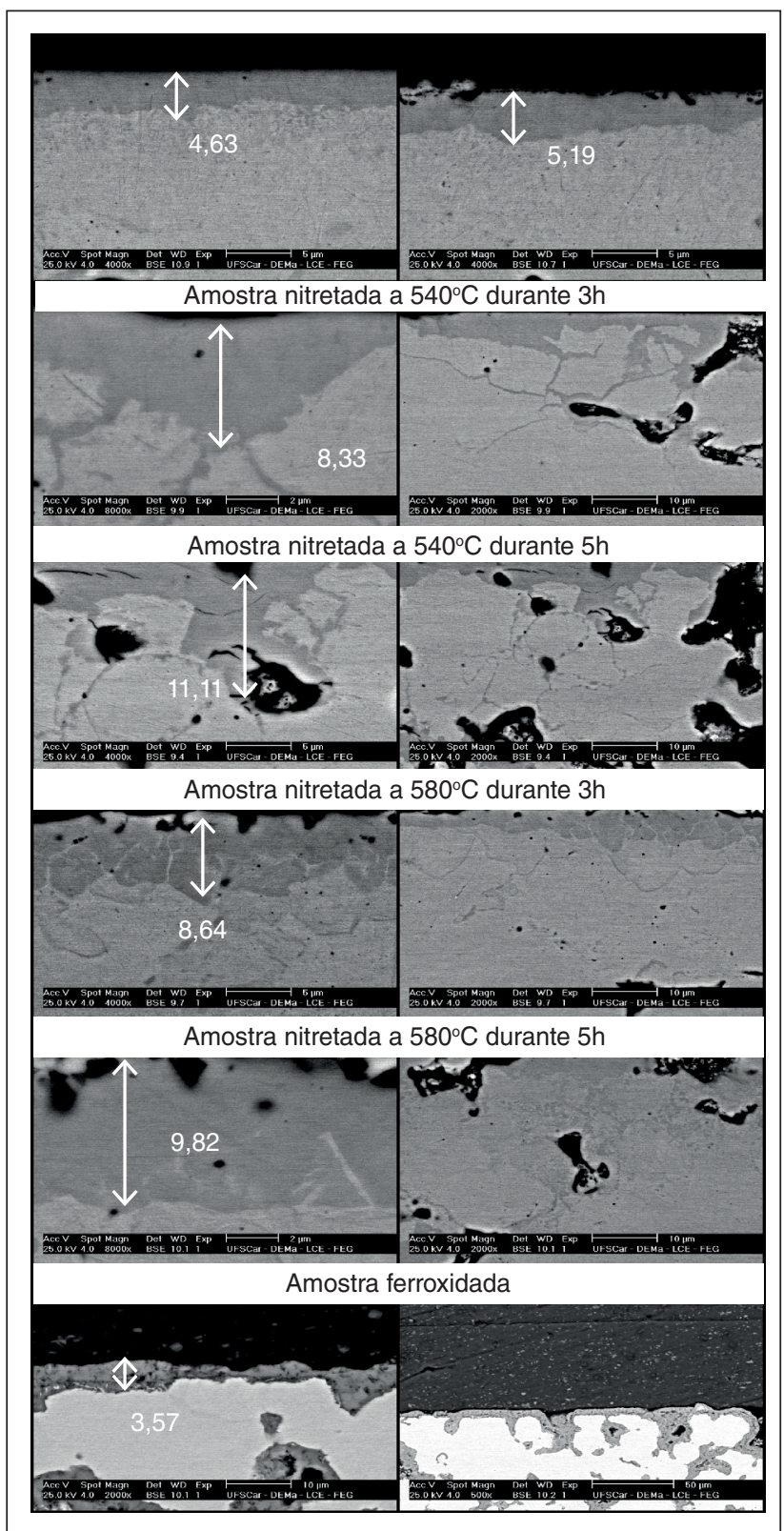

Figura 1: Imagens da análise por MEV da espessura da camada para todas as condições de nitretação, bem como da camada ferroxidada (valores em $\mu \mathrm{m}$ ). núcleo da amostra. A partir das mesmas imagens, gerou-se um gráfico mostrado na Fig. 2 comparando a variação da espessura da camada nitretada com as diversas condições de nitretação, em relação à espessura da camada de ferrox da amostra ferroxidada.

Uma observação a ser feita com relação ao valor de espessura da camada nitretada encontrada é que os valores para as amostras nitretadas por 5 horas foram maiores que as amostras nitretadas por 3 horas, para uma dada temperatura. Em peças sinterizadas onde a porosidade é predominantemente aberta, a espessura da camada, a uma dada temperatura, pode aumentar com o tempo de nitretação ${ }^{(20-22)}$. Pode-se inferir da Fig. 2 que o fator temperatura predomina sobre o fator tempo de nitretação, com relação à espessura da camada nitretada formada. Isto indica que a variação na temperatura de processo é mais significativa que a variação no tempo de nitretação, para o caso de peças originadas da metalurgia do pó.

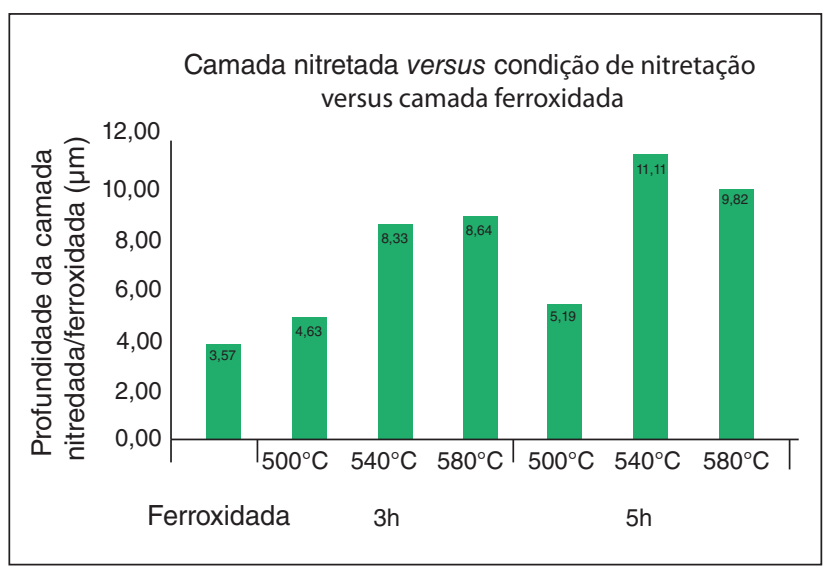

Figura 2: Comparação da espessura da camada nitretada para todas as amostras analisadas, bem como a espessura da camada de ferrox.

\section{Análise via DRX}

A Fig. 3 mostra os difratogramas da amostra de ferro sinterizado sem tratamento nenhum, que apresenta como elemento principal o ferro, e da amostra ferroxidada, identificando a presença de ferro e, principalmente, de óxido de ferro oriundo do processo de ferroxidação. Em particular para a amostra de ferro sinterizado, nota-se picos mais intensos em $2 \theta=44,6^{\circ}$ (110) $100 \%, 2 \theta=65^{\circ}$ (200) $20 \%$ e $2 \theta=82,3^{\circ}$ (211) $30 \%$. Não há alargamento nas linhas do ferro, o que identifica um material bastante cristalino.

A Fig. 4 apresenta os difratogramas das amostras nitretadas em $500^{\circ} \mathrm{C}$ por 3 e 5 horas, onde se nota a presença das fases $\gamma^{\prime}-\mathrm{Fe}_{4} \mathrm{Ne}$ $\mathcal{\varepsilon}-\mathrm{Fe}_{3} \mathrm{~N}$ formadas durante o processo de nitretação.

Para ambos os difratogramas da Fig. 4, são observados picos intensos de ferro, que são devidos provavelmente à pequena espessura da camada nitretada formada, como observada pela análise via MEV.

Nas Figs. 5 e 6 são apresentados os difratogramas das amostras nitretadas a 540 e $580^{\circ} \mathrm{C}$, por 3 e $5 \mathrm{~h}$, respectivamente. Em ambos difratogramas são observados os picos das fases $\gamma^{\prime}-\mathrm{Fe}_{4} \mathrm{~N}$ e $\varepsilon$ - $\mathrm{Fe}_{3} \mathrm{~N}$; também aparecem os picos do metal ferro, porém com intensidades menores. 


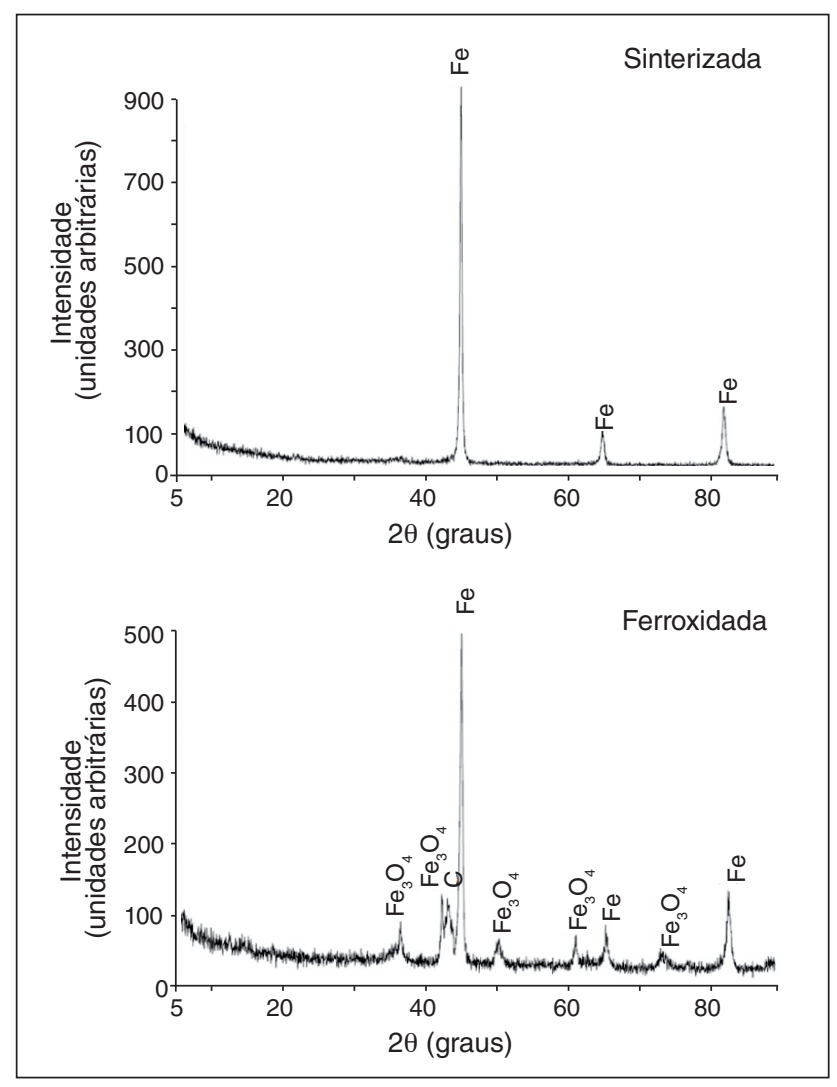

Figura 3: Difratogramas das amostras sinterizada (sem tratamento superficial) e ferroxidada.

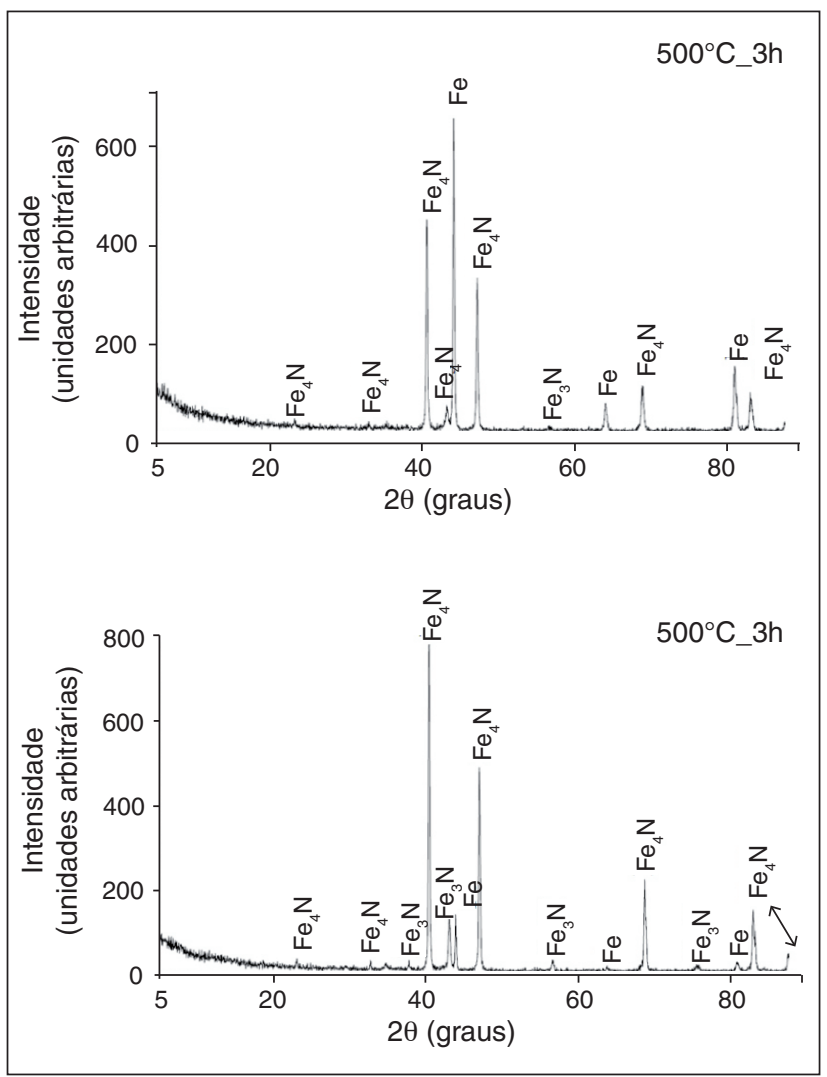

Figura 4: Difratogramas das amostras nitretadas a $500^{\circ} \mathrm{C}$ por $3 \mathrm{e}$ 5 horas.

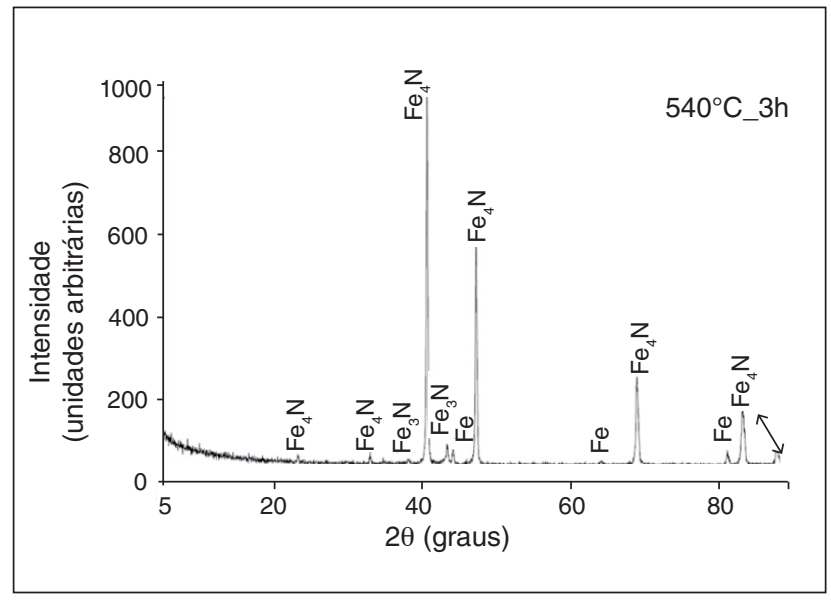

Figura 5: Difratograma da amostra nitretada a $540^{\circ} \mathrm{C}$ por 3 horas.

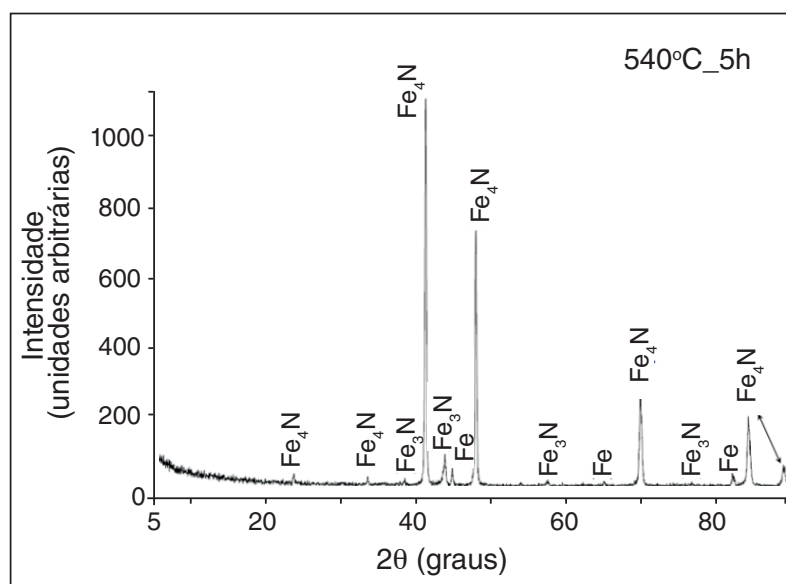

$580^{\circ} \mathrm{C} 3 \mathrm{~h}$
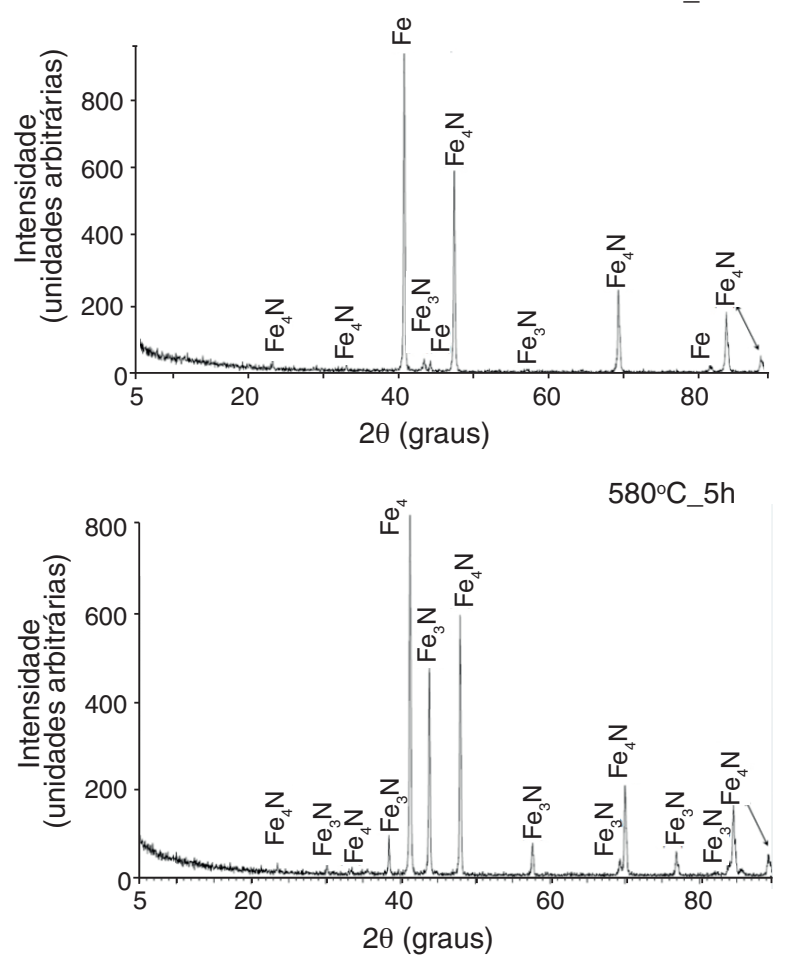

Figura 6: Difratogramas das amostras nitretadas a $540^{\circ} \mathrm{C}$ por 5 horas e a $580^{\circ} \mathrm{C}$ por 3 e 5 horas. 
De modo geral, todas as amostras nitretadas apresentaram a presença das fases $\gamma^{\prime}-\mathrm{Fe}_{4} \mathrm{~N}$ e $\varepsilon$ - $\mathrm{Fe}_{3} \mathrm{~N}$, independente das condições de tratamento empregadas. Os picos mais intensos da fase $\gamma^{\prime}-\mathrm{Fe}_{4} \mathrm{~N}$ foram observados para $2 \theta$ a $41,2^{\circ} ; 48,0^{\circ} ; 71,0^{\circ}$ e $84,5^{\circ}$. Já os picos mais intensos da fase $\varepsilon-\mathrm{Fe}_{3} \mathrm{~N}$ foram observados para $2 \theta$ a $44,0^{\circ}$ e $57,7^{\circ}$. Não foi observado qualquer alargamento nas linhas das fases, indicando a alta cristalinidade dos materiais.

\section{CONCLUSÕES}

Dentre as diversas condições empregadas neste estudo, as camadas mais espessas de nitreto de ferro foram obtidas para as amostras nitretadas por 5 horas, nas temperaturas de 540 e $580^{\circ} \mathrm{C}$, com grande penetração de nitrogênio no interior das amostras. A nitretação a plasma é um processo que está diretamente relacionado à difusão, portanto os aumentos no tempo e, principalmente, na temperatura de tratamento causam um aumento na espessura da camada nitretada. $\mathrm{O}$ fator temperatura predomina sobre o fator tempo de nitretação, quando se espera a obtenção de camadas nitretadas mais espessas. Existe uma pequena diferença na espessura da camada quando se compara os tempos de 3 e 5 horas, porém nada muito significativo quando se compara a espessura em função da temperatura. Isto pode ser explicado devido a camada inicial de nitreto formada inibir a entrada de mais nitrogênio para o interior da amostra. Portanto, o aumento do tempo de nitretação não é o caminho mais favorável para se obter melhores propriedades neste material. A análise via DRX mostrou a presença das fases $\gamma^{\prime}-\mathrm{Fe}_{4} \mathrm{~N}$ e $\varepsilon-\mathrm{Fe}_{3} \mathrm{~N}$ em todas as amostras nitretadas, sendo que para a temperatura de $500^{\circ} \mathrm{C}$ os picos intensos de ferro foram encontrados, devido à pequena espessura da camada formada. Os picos mais intensos de $\gamma^{\prime}-\mathrm{Fe}_{4} \mathrm{~N}$ foram encontrados para as amostras nitretadas em tempos e temperaturas maiores. A formação de $\gamma^{\prime}-\mathrm{Fe}_{4} \mathrm{~N}$ é favorável para altas temperaturas e tempos de nitretação mais longos.

\section{REFERÊNCIAS}

1. Fernandes F. A. P.; Lombardi Neto, A.; Casteletti, L. C.; Oliveira A. M.; Totten, G. E. Heat Treat. Prog. 8 (2008) 41.

2. Michel, H.; Czerwiec, T.; GantoiS, M.; Ablitzer, D.; Ricard, A. Surf. Coat. Technol. 72 (1995) 103.

3. Alves Jr., C. Fenomenologia da nitretação iônica: fundamentos e aplicações. Natal, EDUFRN, 2001.

4. Cocke, D.L.; Jurckkrajman, M.; Veprek, S. J. Electrochem. Soc. 136 (1986) 3655.

5. Pizzolatti, J.; Elbern, A. W. In: Anais do $8^{\circ}$ Congresso Brasileiro de Engenharia e Ciências dos Materiais, Campinas, pp 113-116, 1988.

6. Gontijo, L.C.; Machado; R., Miola, E.J.; Casteletti, L.C.; Nascente, P.A.P. Surf. Coat. Technol. 183 (2004) 10.

7. Metin, E; Inal, O.T. J. Mater. Sci. 22 91987) 2783.
8. Salas, O; Figueroa, F.; Palacios, M.; Oseguera, J. Surf. Coat. Technol. 86-87 (1996) 332.

9. Totten, G. E.; Howes, M. A. H. Steel Heat Treatment Handbook. 1st ed., Marcel Dekker, New York, 1997.

10. Miola, E.J.; de Sousa, S.D.; Nascente, P.A.P.; Olzon-Dionysio, M.; Olivieri, C.A.: Spinelli, D., J. Vac. Sci. Technol. A 18 (2000) 2733.

11. Muñoz Riofano, R.M.; Casteletti, L.C.; Nascente, P.A.P. Surf Coat. Technol. 200 (2006) 6101.

12. Collins, G.A.; Hutchings, R.; Tendys, J.; J. Mater. Sci. Eng. A 139 (1991) 171

13. Collins, G.A.; Hutchings, R.; Short, K.T.; Tendys, J.; Van Der Valk, S.H. Surf. Coat. Technol. 84 (1996) 537.

14. Borgioli, F.; Fossati, A.; Galvanetto, E.; Bacci, T. Surf. Coat. Technol. 200 (2005) 2474

15. Borgioli, F.; Fossati, A.; Galvanetto, E.; Bacci, T.; Pradelli, G. Surf Coat. Technol. 200 (2005) 5505.

16. Hume-Rothery, W. Estrutura das ligas de ferro: introdução elementar. Edgard Blucher, São Paulo, 1968.

17. Kittel, C. Introduction to solid state physics. $5^{\text {a }}$. Ed., Jonh Wiley \& Sons, New York, 1976

18. Reed-Hill, R. E. Princípios de metalurgia física. $2^{\mathrm{a}}$. Ed., Guanabara Dois, Rio de Janeiro, 1982.

19. Fernandes, F.A.P.; Heck, S.C.; Pereira, R.G.; Picon, C.A.; Nascente, P.A.P.; Casteletti, L.C. Surf. Coat. Technol. 204 (2010) 3087.

20. Hoffmann, F.T.; Mayr, P. ASM Handbook, vol. 18, ASM International, New York, 1992

21. Maliska, A.M.; Menezes, A.; Angioletto, E.; Pavanati, H.C.; Drago, V.; Muzart, J.L.; Klein, A.N. In: Anais do $13^{\circ}$ Congresso Brasileiro de Engenharia e Ciências dos Materiais, Curitiba, pp. 16451655, 1998.

22. Maliska, A.M.; Pavanati, H.C.; Klein, A.N.;Muzart, J.L. Mater. Sci. Eng. A 352 (2003) 273.

23. J. E. Harry, Introduction to Plasma Technology (Wiley- $\mathrm{VCH}$ Berlin, 2010)

24. A. Fridman, Plasma Chemistry (Cambridge University Press, USA, 2008)

25. R. d'Agostino, P. Favia, Y. Kawai, H. Ikegami, N. Sato, F. ArefiKonsari (eds.) Advanced Plasma Technology (Wiley-VCH Berlin, 2008)

26. R. Hippler, S. Pfau, M. Schmidt, K. Schoenbach (eds.) in Low Temperature Plasma Physics - Fundamental Aspects and Applications, vols.1 and 2, 2nd edn. (Wiley-VCH, Berlin, 2008)

27. H. Biederman (ed.), Plasma Polymer Films (Imperial College Press, London, 2004)

28. P. K. Chu, J. Y. Chen, L. P. Wang. N. Huang, Mater. Sci. and Eng R 36, $143(2002)$

29. K. Ostrikov, Plasma Nanoscience (Wiley-VCH, Berlin, 2008)

30. K. Ostrikov, S. Xu, Plasma-Aided Nanofabrication - From Plasma Sources to Nanoassembly (Wiley-VCH, Berlin, 2007)

31. G. Cicala, M. Creatore, P. Favia, R. Lamendola and R. d'Agostino, App. Phys. Lett., 75 (1999) 37

32. P. Favia and R. d'Agostino, Surf. Coat. Technol., 98 (1998) 1102 
33. H. Biederman and D. Slavínská, Surf. Coat. Technol., 125 (2000) 371

34. R. Itatani, Plasm. and Ions, 1 (1998) 37

35. S. K. Hendricks, K. Kwok, M. Shen, T. A. Horbett, B. D. Ratner and J. D. Bryers, J. Biomed. Mater. Res., 50 (2000) 160

36. M. N. Mar, B. D. Ratner and S. S. Yee, Sensors and Actuators B, 54 (1999) 125
37. C. S. Kwok, T. A. Horbett and B. D. Ratner, J. Control. Release, 62, (1999) 301

38. G. P. Lopez, B. D. Ratner, C. D. Tidwell, C. L. Haycox, R. J. Rapoza and T. A. Horbett, J. Biomed. Mater. Res., 26 (1992) 415

39. C. Oehr, M. Müller, B. Elkin, D. Hegemann and U. Vohrer, Surf. Coat. Technol., 116-119 (1999) 25 